\title{
ISSR and AFLP Markers Differ among American Watermelon Cultivars with Limited Genetic Diversity
}

\author{
Amnon Levi ${ }^{1}$ and Claude E. Thomas \\ U.S. Department of Agriculture, Agricultural Research Service, U.S. Vegetable Laboratory, 2700 \\ Savannah Highway, Charleston, SC 29414 \\ M. Newman \\ U.S.Department of Agriculture, Agricultural Research Service, Plant Genetic Resources and Conservation \\ Unit, 1109 Experiment Street, Griffin, GA 30223-1797
}

O.U. K. Reddy

Center of Plant Biotechnology and Genomics, Alcorn State University, Lorman, MS 39096

X. Zhang

Syngenta Seeds, 21345 Road 98, Woodland, CA 95695

Y. Xu

National Engineering Research Center for Vegetables, Beijing 100089, China

ADDITIONAL INDEX wORDs. RAPD, DNA markers, germplasm evaluation, vegetable breeding, Citrullus lanatus

\begin{abstract}
Wide phenotypic diversity exists among American heirloom cultivars of watermelon (Citrullus lanatus var. lanatus). However, in published studies, low or no polymorphism was revealed among those heirlooms using isozyme or randomly amplified polymorphic DNA (RAPD) markers. In this study, experiments with inter-simple sequence repeat (ISSR) [also known as simple sequence repeat-(SSR-) anchored primers] and amplified fragment-length polymorphism (AFLP) markers produced high polymorphisms among watermelon heirloom cultivars. ISSR (111) and AFLP (118) markers (229 total) identified $80.2 \%$ to $97.8 \%$ genetic similarity among heirloom cultivars. The phylogenetic relations based on ISSR and AFLP markers are highly consistent with the parental records available for some of the heirloom cultivars, providing confidence in the dendogram constructed for heirlooms based on similarity values. As compared with RAPD markers, ISSRs and AFLPs are highly effective in differentiating among watermelon cultivars or elite lines with limited genetic diversity.
\end{abstract}

Watermelon is grown in 44 states in the United States. The largest production areas are in Florida, California, Texas, Georgia, and Arizona. U.S. watermelon production has increased from 1.2 M tons in 1980 to $3.9 \mathrm{M}$ tons in 2002 with a farm value of $\$ 291$ million in 2002 [U.S. Dept. of Agriculture (USDA), 2003]. In recent years, there has been an increased demand by consumers for seedless (triploid) watermelons. Consequently, production of triploid watermelons has increased significantly. During 2002, $>82 \%$ and $51 \%$ of the watermelons produced in California and in the Southeast of the United States (including Virginia, North Carolina, South Carolina, Georgia, and Florida), respectively, were seedless (USDA, 2003). There is a continuous need to develop new seedless watermelon cultivars suitable to consumer needs.

A large number of American heirloom cultivars are kept at the USDA, ARS, National Seed Storage Laboratory, Fort Collins, Colo. (Germplasm Resources Information Network; http://www. ars-grin.gov/cgi-bin/npgs/html/croplist.pl). These cultivars vary in fruit quality (fruit shape, size, flesh color and texture, sugar, and carotenoid content) and can be useful for further develop-

Received for publication 3 Sept. 2003. Accepted for publication 26 Jan. 2003. The authors gratefully acknowledge Laura Pence for technical assistance and Richard Fery for reviewing the manuscript. The use of trade names in this publication does not imply endorsement by the USDA of the products named, or criticism of similar ones not mentioned.

${ }^{1}$ Corresponding author; e-mail alevi@saa.ars.usda.gov ment of elite watermelon cultivars, particularly triploid seedless watermelons. However, there is insufficient information regarding phylogenetic relationships among many of those heirlooms (Elmstrom, 1999). Furthermore, although there is wide phenotypic diversity among watermelon heirloom cultivars, most of them appeared to have a narrow genetic background reflected in a large number of monomorphic and a few polymorphic RAPD markers among cultivars (Levi et al., 2001; Zhang et al., 1994). Thus, it is vital for breeders and researchers to employ in their breeding programs highly polymorphic DNA markers that accurately differentiate among closely related watermelon cultivars and breeding lines.

The objectives of this study were to 1) determine whether inter-simple sequence repeat (ISSR) and amplified fragment-length polymorphism (AFLP) markers detect higher polymorphism among watermelon cultivars as compared to randomly amplified polymorphic DNA (RAPD) markers, 2) construct a dendrogram based on AFLP and ISSR markers that delineate phylogenetic relationships among watermelon heirlooms with limited genetic diversity, 3) determine to what degree the phylogenetic relationships based on ISSR and AFLP markers are consistent with the parental records provided for watermelon (Elmstrom, 1999), and 4) construct a DNA fingerprinting table based on polymorphic ISSR and AFLP markers that can be useful in differentiating among heirloom cultivars or elite watermelon lines. 


\section{Material and Methods}

Plant material and DNA isolation. Seeds of 44 heirloom watermelon cultivars were obtained from various sources (Table 1) and germinated in the greenhouse $\left(26^{\circ} \mathrm{C}\right.$ day $/ 20^{\circ} \mathrm{C}$ night temperatures). Young leaves were collected from four to five plants ( 3 weeks old) of each of the cultivars and stored at $-80{ }^{\circ} \mathrm{C}$. To avoid co-isolation of polysaccharides, polyphenols, and other secondary compounds that damage DNA, we used an improved procedure for isolation of DNA from young leaves of watermelon (Levi and Thomas, 1999; Levi et al., 2001).

DNA AMPLIFICATION CONDITIONS USING ISSR PRIMERS AND GEL ELECTROPHORESIS. ISSR primers (15 to 20 decamer oligonucleotides) were purchased from the Univ. of British Columbia, Vancouver, B.C., Canada (primer 800-899). Polymerase chain reactions (PCR) were performed in $25 \mu \mathrm{L}$ reaction buffer containing $20 \mu \mathrm{M} \mathrm{NaCl}, 50 \mathrm{~mm}$ Tris-HCl pH 9, 1\% Triton-X-100, 0.01\% gelatin, $1.6 \mathrm{~mm} \mathrm{MgCl}_{2}, 200 \mu \mathrm{M}$ each of dATP, dCTP, dGTP, and dTTP (Sigma), 0.2 mm primer, 7 units Taq DNA Polymerase (Promega, Madison, Wis., supplied in storage buffer A), and 15 ng template DNA (Levi et al., 1993). Amplification reactions were carried out for 45 cycles in a PTC-200 thermocycler (MJ Research, Watertown, Mass.), programmed for $60 \mathrm{~s}$ to denature DNA at $92{ }^{\circ} \mathrm{C}, 70 \mathrm{~s}$ for DNA annealing at 48 to $62^{\circ} \mathrm{C}$ (Table 2), and $120 \mathrm{~s}$ for DNA transcription at $72^{\circ} \mathrm{C}$. The DNA annealing temperature was optimized for each primer based on its meltingtemperature (Tm) (Table 2). Amplification products were separated by electrophoresis in $1.4 \%$ agarose gels in $0.5 \times$ Tris-borate buffer (Sambrook et al., 1989). The gels were stained with 0.5 $\mu \mathrm{g} \cdot \mathrm{mL}^{-1}$ ethidium bromide solution for $30 \mathrm{~min}$ and destained for $15 \mathrm{~min}$ in distilled water. DNA fragments were visualized under UV light and photographed using a still video system (Gel Doc 2000; Bio-Rad, Hercules, Calif.). The molecular weights of the amplification products were calculated using 1-kb DNA ladder standards (Gibco BRL, Gaithersburg, Md.).

Table 1. Watermelon heirlooms examined for genetic similarity using AFLP and ISSR markers.

\begin{tabular}{|c|c|c|c|c|}
\hline Cultivar & $\begin{array}{c}\text { Source of } \\
\text { seeds }\end{array}$ & $\begin{array}{c}\text { Year } \\
\text { Released }\end{array}$ & Breeding Parentage & $\begin{array}{l}\text { Closest Cultivars on Dendogram (Figure 2) } \\
\text { and their similarity value }(\%) \text { with heirloom }\end{array}$ \\
\hline Allsweet & Sunseeds ${ }^{2}$ & 1972 & [Miles $\times$ Peacock] $\times$ Charleston Gray & Calhoun Gray (96.4\%) \\
\hline AU-Golden Producer & Hollar ${ }^{y}$ & 1993 & A selection from $\mathrm{AU}$-Producer & AU-Producer $(93.0 \%)$ \\
\hline AU-Jubilant & Hollar & 1985 & Jubilee $\times$ PI 271778 & Black Diamond (93.4\%) \\
\hline AU-Producer & Hollar & 1985 & Crimson Sweet x PI 189225 & AU-Golden Producer (93.0\%) \\
\hline Black Diamond & Sunseeds & $\ldots T$ & -- & Blackstone $(94.4 \%)$ \\
\hline Blackstone & Hollar & - & Black Diamond, Fairfax & Black Diamond (94.4\%) \\
\hline Calhoun Gray & Sunseeds & $\cdots$ & -- & Allsweet $(96.4 \%)$, CalSweet (93.9\%) \\
\hline CalSweet & Sunseeds & -- & -- & Calhoun Gray (93.9\%), Family Fun (93.4\%) \\
\hline Charleston Gray & NSL- $5267^{x}$ & 1954 & Africa 8, lowa Belle,Garrison, Hawkesbury, Leesburg & Dixielee $(90.6 \%)$ \\
\hline Coles Early & Sunseeds & 1892 & 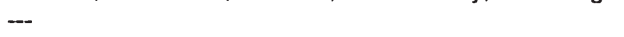 & Dixie-Queen (83.7\%), Fairfax (80.2\%) \\
\hline Congo & Syngenta $^{\mathrm{W}}$ & 1949 & (African $\times$ low Belle) $\times$ Garrison & Blackstone $(90.8 \%)$ \\
\hline Crimson Sweet & Hollar & 1963 & (Miles $\times$ Peacock) $\times$ Charleston Gray & AU-Producer (89.9\%), Garrison (88.4\%) \\
\hline Dixielee & Hollar & 1979 & Texas W5, Wilt resistant Peacock, Fairfax, Summit & Family Fun (91.5\%), Charleston Gray $(90.6 \%)$ \\
\hline $\begin{array}{l}\text { Dixie Queen } \\
\text { Dunbarton }\end{array}$ & $\begin{array}{l}\text { Sunseeds } \\
\text { NSL-6637 }\end{array}$ & $\begin{array}{l}1890 \\
1953\end{array}$ & $\begin{array}{l}\text { F1 Hybrid -- } \\
\text { (African } \times \text { lowa Belle) } \times \text { Garrison }\end{array}$ & $\begin{array}{l}\text { Jubilee }(92.5 \%) \\
\text { Klondike-Strip }(89.1 \%)\end{array}$ \\
\hline $\begin{array}{l}\text { Fairfax } \\
\text { Family Fun }\end{array}$ & $\begin{array}{l}\text { Sunseeds } \\
\text { Syngenta }\end{array}$ & $\begin{array}{l}1952 \\
1973\end{array}$ & $\begin{array}{l}\text { Garrison, African, lowa Belle, Leesburg, Hawkesbury } \\
\text { F1 Hybrid --- }\end{array}$ & $\begin{array}{l}\text { Garrisonian }(85.3 \%) \text {, Coles Early }(83.7 \%) \\
\text { CalSweet }(93.4 \%) \text {, Dixielee }(91.5 \%)\end{array}$ \\
\hline Garrison & NSL-2053 & --- & $-\ldots$ & Garrisonian (92.5\%), Crimson Sweet (88.4\%) \\
\hline Garrisonian & Willhite $^{v}$ & 1957 & Africa 8, lowa Belle, Garrison, Hawkesbury, Leesburg & Garrison $(92.5 \%)$ \\
\hline Georgia Rattlesnake & Seed Savers" & -.. & - & Leesburg $(90.6 \%)$ \\
\hline Golden Honey & Hollar & 1954 & Mixed Strain from Japan Seed, Co. & Hawkesbury (89.3\%) \\
\hline Golden Midget & NSL-5288 & 1959 & New Hampshire Midget $x$ Pumpkin Rind & Mickylee $(92.3 \%)$ \\
\hline Hawkesbury & Syngenta & 1936 & -- & Golden Honey (89.3\%) \\
\hline lopride & Syngenta & $\ldots$ & - & Kleckely's Sweet (89.4\%) \\
\hline Ironsides & NSL-7369 & 1950 & [Leesburg $\times$ Hawkesbury] $\times$ Garrison & New Hampshire Midget (85.6\%) \\
\hline Jubilee & Hollar & 1963 & Africa 8 , lowa Belle, Garrison, Hawkesbrry, Leesburg & Dixie Queen (92.5\%), Georgia Rattlesnake (90.1\%) \\
\hline King and Queen & Hollar & $\ldots$ & - & Leesburg (88.6\%), lopride $(87.4 \%)$ \\
\hline Kleckely's Sweet & Seed Savers & --- & -- & Miles $(91.8 \%)$ \\
\hline Klondike & Syngenta & 1959 & - & Dunbarton $(89.1 \%)$ \\
\hline Leesburg & NSL-7368 & 1936 & Selection from Kleckley Sweet & Georgia Rattlesnake $(90.6 \%)$ \\
\hline Mickylee & Hollar & 1986 & Texas W5, Fairfax, Summit, Graybelle & Minilee (94.6\%), Golden Midget (92.3\%) \\
\hline Miles & NSL-6688 & 1948 & Dixie Queen x Klondike R-7 & Parker $(96.2 \%)$ \\
\hline Minilee & Hollar & 1986 & Texas W5, Fairfax, Summit, Graybelle & Mickylee $(94.6 \%)$ \\
\hline New Hampshire Midget & Syngenta & 1951 & Favorite Honey $x$ Dakota Sweet & Minilee $(90.8 \%)$ \\
\hline Northern Sweet & Syngenta & 1932 & $\ldots$ & Summit $(92.9 \%)$. Picnic $(92.6 \%)$ \\
\hline Parker & Willhite & --- & F1 Hybrid -.- & Miles $(96.2 \%)$, Summit $(92.9 \%)$ \\
\hline Peacock & Hollar & 1939 & -- & Sweet Princes $(92.4 \%)$, Prince Charles $(90.6 \%)$ \\
\hline Picnic & Syngenta & 1972 & Peacock type & Prince Charles (97.7\%), Northern Sweet $(92.6 \%)$ \\
\hline Prince Charles & Hollar & 1978 & F1 Hybrid -.- & Picnic $(97.7 \%)$ \\
\hline Sugar Baby & Sunseeds & 1955 & Tough Sweets selection & Klondike-Strip (88.7\%) \\
\hline Stone Mountain & Hollar & 1924 & -- & Stone Mountain \#5 (89.8\%). Sweet Princes (86.9\%) \\
\hline Stone Mountain \#5 & Syngenta & 1936 & Stone mountain $\times$ lowa Belle & Stone Mountain (89.8\%) \\
\hline
\end{tabular}

${ }^{Z}$ Sunseeds Co. (Acampo, California). 'Hollar Seeds, Inc. (Rocky Ford, Colorado). ${ }^{X}$ Accession number provided for each of the heirloom cultivars kept at the USDA National Seed Storage Laboratory (Fort Collins, Colorado). "'Syngenta Seeds, Inc. (Napels, Florida). 'Willhite Seed, Inc. (Poolville, Texas). "Seed Savers Exchange (Decorah, lowa). Tinformation unavailable. 
Table 2. The nucleotide sequence of ISSR primers, the optimal annealing temperature in PCR reactions, and the number of markers produced by each primer (number of polymorphic markers in parenthesize).

\begin{tabular}{|c|c|c|c|}
\hline Primer & Sequence & $\begin{array}{l}\text { Annealing } \\
\text { temp }\left({ }^{\circ} \mathrm{C}\right)\end{array}$ & $\begin{array}{c}\text { No. of } \\
\text { markers }\end{array}$ \\
\hline$\overline{808}$ & AGAGAGAGAGAGAGAGC & 59 & $5(4)$ \\
\hline 809 & GAGGAGAGAGAGAGAGG & 59 & $4(3)$ \\
\hline 810 & GAGAGAGAGAGAGAGAT & 53 & $5(3)$ \\
\hline 812 & GAGAGAGAGAGAGAGAA & 53 & $2(1)$ \\
\hline 813 & СТСТСТСТСТСТСТСТТ & 53 & $1(1)$ \\
\hline 815 & СТСТСТСТстстстСТG & 48 & $1(1)$ \\
\hline 816 & CACACACACACACACAT & 54 & $2(1)$ \\
\hline 817 & CACACACACACACACAA & 54 & $2(1)$ \\
\hline 818 & CACACACACACACACAG & 54 & $4(3)$ \\
\hline 820 & GTGTGTGTGTGTGTGTC & 59 & $4(2)$ \\
\hline 821 & GTGTGTGTGTGTGTGTT & 59 & $2(0)$ \\
\hline 822 & ТСТСТСТСТСТСТСТСА & 54 & $2(0)$ \\
\hline 823 & ТСТСТСТСТСТСТСТСС & 54 & $6(2)$ \\
\hline 824 & ТСТСТСТСТСТСТСТСG & 54 & $2(0)$ \\
\hline 825 & ACACACACACACACACT & 54 & $6(2)$ \\
\hline 826 & ACACACACACACACACC & 62 & $4(4)$ \\
\hline 828 & TGTGTGTGTGTGTGTGA & 54 & $3(0)$ \\
\hline 829 & TGTGTGTGTGTGTGTGC & 62 & $1(0)$ \\
\hline 834 & AGAGAGAGAGAGAGAGYT & 59 & $4(4)$ \\
\hline 835 & AGAGAGAGAGAGAGAGYC & 59 & $5(2)$ \\
\hline 836 & AGAGAGAGAGAGAGAGYA & 54 & $1(1)$ \\
\hline 840 & GAGAGAGAGAGAGAGAYT & 54 & $4(4)$ \\
\hline 841 & GAGAGAGAGAGAGAGAYC & 54 & $3(1)$ \\
\hline 842 & GAGAGAGAGAGAGAGAYG & 59 & $2(2)$ \\
\hline 843 & CTCTCTCTCTCTCTCTRA & 54 & $3(3)$ \\
\hline 844 & CTCTCTCTCTCTCTCTRC & 54 & $3(3)$ \\
\hline 845 & СTCTCTCTCTCTCTCTRG & 54 & $3(0)$ \\
\hline 847 & CACACACACACACACAAGC & 62 & $2(2)$ \\
\hline 849 & GTGTGTGTGTGTGTGTCTA & 54 & $3(2)$ \\
\hline 851 & GTGTGTGTGTGTGTGTCTG & 62 & $1(0)$ \\
\hline 852 & ТСТСТСТСТСТСТСТСAGA & 54 & $3(3)$ \\
\hline 853 & ТСТСТСТСТСТСТСТСАGT & 54 & $2(1)$ \\
\hline 854 & тСТСТСТСТСТСТСТСAGG & 54 & $1(1)$ \\
\hline 856 & ACACACACACACACACCTA & 62 & $2(1)$ \\
\hline 864 & ATGATGATGATGATGATG & 54 & $5(5)$ \\
\hline 880 & GGAGAGGAGAGGAGA & 54 & $4(4)$ \\
\hline 889 & AGTCGTAGTACACACACACACAC & 62 & $5(3)$ \\
\hline 891 & ACTACGACTTGTGTGTGTGTGTG & 62 & $2(2)$ \\
\hline
\end{tabular}

AFLP PROCEDURE. The AFLP procedure developed by Vos et al. (1995) was modified using a commercially available kit (Plant Mapping Kit-RegularPlantGenome; Applied Biosystems, Foster City, Calif.). According to the manufacturer's protocol, a highquality genomic DNA (500 ng; intact and with a 260/280 O.D. ratio of 1.8) of each cultivar was added to a restriction-ligation mixture containing EcoRI (5 U), MseI (1 U), T4 ligase (1 U), $1 \times$ T4 ligase buffer (New England BioLabs, Beverly, Mass.), 50 $\mathrm{ng} \cdot \mu \mathrm{L}^{-1} \mathrm{BSA}$, and $50 \mathrm{mHm} \mathrm{NaCl}$. Additionally, MseI and EcoRI adapters (20 and $2 \mathrm{~nm} \cdot \mu \mathrm{L}^{-1}$, respectively), and sterile distilled water were added to bring the mixture to a final volume of $11 \mu \mathrm{L}$ (New England BioLabs). Following $2 \mathrm{~h}$ incubation at $37^{\circ} \mathrm{C}$, the restriction-ligation reaction mix was diluted 18 -fold by adding $189 \mu \mathrm{L}$ purified water.

Each preselective PCR amplification contained $4 \mu \mathrm{L}$ of the diluted restriction-ligation reaction, $1 \mu \mathrm{L}$ of EcoRI / MseI primer solution, and $15 \mu$ LAFLPCore Mix (Plant Mapping Kit-Regular Plant Genome, Applied Biosystems). All PCRs were performed in a PTC 200 thermocycler (MJ Research, Watertown, Mass.). Preselective PCR amplifications were carried through 20 cycles, each consisting of $20 \mathrm{~s}$ to denature DNA at $94{ }^{\circ} \mathrm{C}, 30 \mathrm{~s}$ for DNA annealing at $56^{\circ} \mathrm{C}$, and $120 \mathrm{~s}$ for DNA transcription at $72^{\circ} \mathrm{C}$. Then, the final cycle included $30 \mathrm{~min}$ incubation at $60{ }^{\circ} \mathrm{C}$ to complete any DNA annealing and transcription, and cooling at $4{ }^{\circ} \mathrm{C}$. Each of the $20 \mu \mathrm{L}$ of preselective amplification reactions were diluted 10 -fold by adding $180 \mu \mathrm{L}$ sterile distilled water.

For selective amplification, $1.5 \mu \mathrm{L}$ of diluted reaction was mixed with one of the following selective primer sets: pair 1) 5'GAATTC $(E c o R I)-\mathrm{AAG}$ and 5'TTAA $(M s e I)$-CAT, pair 2) 5'GAATTC $(E c o R I)-A A G$ and 5'TTAA $(M s e I)-C T C$, pair 3) 5'GAATTC (EcoRI)-ACC and 5'TTAA (MseI)-CAT, and pair 4) 5'GAATTC $(E c o R I)-A C C$ and 5'TTAA(MseI)-CAA according to manufacturer's instructions (Plant Mapping Kit-Regular Plant Genome; Applied Biosystems). The first part of the selective amplification reaction consisted of a cycle of $140 \mathrm{~s}$ at $94{ }^{\circ} \mathrm{C}$ to denature DNA, $30 \mathrm{~s}$ for DNA annealing at $65^{\circ} \mathrm{C}$, and $120 \mathrm{~s}$ 


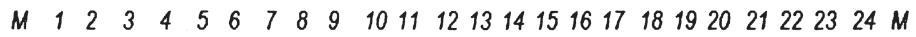

UBC-844

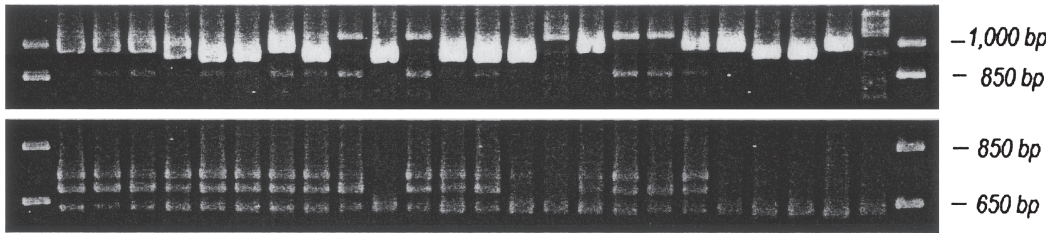

Fig. 1. PCR-ISSR patterns (on $1.4 \%$ agarose-gel) of watermelon cultivars produced by ISSR primers UBC844, and UBC-849. Lanes are: M) 100 bp DNA ladder markers (Life Technologies, Rockville, Maryland), 1) 'Allsweet', 2) 'Picnic', 3) 'AU-Golden Producer', 4) 'AU-Jubilant', 5) 'AU-Producer', 6) 'Blackstone', 8) 'Calhoun Gray', 9) 'Calsweet', 10) 'Charleston Gray' (NSSL), 11) 'Charleston Gray'(NKLawn\& Garden Co), 12) 'Coles Early', 13) 'Congo', 14) 'Crimson Sweet', 15) 'Dixielee', 16) 'Dixie Queen', 17) 'Dunbarton', 18) 'Fairfax', 19) 'Family Fun', 20) 'Garrison', 21) 'Garrisonian', 22) 'Georgia Rattlesnake', 23) 'Golden Honey', and 24) 'Golden Midget'.

Table 3. ISSR and AFLP markers polymorphic among American heirloom cultivars: Allsweet (AS), AU-Jubille (AJ), AU-Golden Producer (AG), AU- Producer (AP), Black Diamond (BD), Blackstone (BS), CalSweet (CS), Calhoun Gray (CG), Charleston Gray (CY), Coles Early (CE), Congo (CO), Crimson Sweet (CT), Dixielee (DL), Dixie Queen (DQ), Dunbarton (DB), Fairfax (FX), Family Fun (FF), Garrison (GR), Garrisonian (GN), Georgia-Rattlesnake (GS), Golden Honey (GH), Golden Midget (GM), Hawkesbury (HU), Iopride (IP), Ironsides (IS), Jubilee (JB), King \& Queen (KQ), Kleckely's Sweet (KS), Klondike (KD), Leesburg (LB), Mickylee (MK), Miles (MS), Minilee (MN), New Hampshire Midget (NM), Northern Sweet (NS), Parker (PK), Peacock (PO), Picnic (PI), Prince Charles (PC), Stone Mountain (SM), Stone mountain \#5 (S5), Sugar Baby (SB), Summit (SU), and Sweet Princes (SP).

\begin{tabular}{|c|c|c|c|c|c|c|c|c|c|c|c|c|c|c|c|c|c|c|c|c|c|c|c|c|c|c|c|c|c|c|c|c|c|c|c|c|}
\hline$\overline{S R}$ & & & & & & & & & & & & & & & & & & & & & & & & & & & & & & & & & & & & \\
\hline darkers & & & & & & & & & & & & & & & & & & & & & & & & & & & & & & & & & & & & \\
\hline $808-700^{2}$ & 1 & 1 & 1 & 1 & 1 & 1 & 1 & 1 & 0 & 1 & 0 & 0 & 0 & 1 & 1 & 0 & 1 & 0 & 0 & 1 & 1 & 1 & 1 & & 1 & 1 & 1 & 1 & 1 & 1 & 1 & 1 & 1 & 1 & 1 & 1 \\
\hline $808-675$ & 1 & 1 & 1 & 1 & 1 & 1 & 1 & 1 & 1 & 1 & 1 & 0 & 1 & 1 & 1 & 0 & 1 & 0 & 0 & 1 & 1 & 1 & 1 & 1 & 1 & 1 & 1 & 1 & 1 & 1 & 1 & 1 & 1 & 1 & 1 & 1 \\
\hline 808-525 & 1 & 1 & 1 & 1 & 1 & 1 & 1 & 1 & 1 & 0 & 1 & 1 & 0 & 1 & 1 & 1 & 0 & 0 & 0 & 1 & 1 & 0 & 1 & 1 & 1 & 1 & 0 & 1 & 0 & 1 & 0 & 0 & 0 & 0 & 1 & 1 \\
\hline 808-375 & 1 & 1 & 1 & 1 & 1 & 1 & 1 & 1 & 1 & 1 & 1 & 1 & 1 & 1 & 0 & 1 & 1 & 1 & 1 & 1 & 0 & 0 & 1 & 1 & & 1 & & 0 & 0 & 1 & 0 & 1 & 0 & 0 & 1 & 1 \\
\hline $809-2000$ & 1 & 0 & 0 & 0 & 1 & 0 & 0 & 0 & 0 & 0 & 0 & 0 & 1 & 0 & 1 & 0 & 0 & 0 & 0 & 0 & 0 & 1 & 0 & 0 & 0 & 0 & 0 & 0 & 0 & 0 & 1 & 1 & 1 & 1 & 0 & 1 \\
\hline $809-875$ & 1 & 0 & 0 & 0 & 1 & 0 & 0 & 1 & 0 & 0 & 0 & 0 & 1 & 0 & 0 & 0 & 0 & 0 & 0 & 0 & 0 & 1 & 0 & 0 & 0 & 0 & 0 & 0 & 0 & 0 & 1 & 1 & 1 & 1 & 0 & 1 \\
\hline $9-450$ & 0 & 0 & 0 & 0 & 0 & 0 & 1 & 0 & 0 & 0 & 0 & 0 & 0 & 0 & 0 & 0 & 1 & 0 & 0 & 0 & 0 & 1 & 0 & 0 & 0 & 0 & 0 & 0 & 1 & 0 & $i$ & 0 & 1 & 1 & 1 & 0 \\
\hline $810-1350$ & 0 & 1 & 1 & 1 & 0 & 0 & 0 & 0 & 1 & 1 & 0 & 0 & 0 & 1 & 1 & 0 & 0 & 0 & 0 & 1 & 1 & 1 & 1 & 1 & 1 & 1 & 1 & 1 & 1 & 1 & 1 & 1 & 1 & 1 & 1 & 1 \\
\hline $810-600$ & 1 & 1 & 1 & 1 & 1 & 0 & 0 & 0 & 0 & 1 & 1 & 1 & 1 & 1 & 1 & 1 & 0 & 0 & 0 & 1 & 1 & 1 & 1 & 0 & & 0 & 1 & 0 & 9 & 0 & 0 & 0 & 0 & 1 & 1 & 0 \\
\hline $5-800$ & 1 & 1 & 1 & 1 & 1 & 1 & 1 & 1 & 1 & 0 & 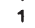 & 0 & 1 & 0 & 0 & 0 & 1 & 1 & 1 & 0 & 0 & 0 & 0 & 0 & 0 & 0 & 1 & 0 & 0 & 0 & 1 & 0 & 0 & 0 & 0 & 0 \\
\hline $818-625$ & 1 & 1 & 1 & 1 & 1 & 1 & 1 & 1 & 1 & 0 & 1 & 1 & 1 & 0 & 0 & 0 & 1 & 1 & 1 & 0 & 0 & 0 & 0 & & 0 & 0 & 0 & 0 & 0 & 0 & 0 & 0 & 0 & 0 & 0 & 0 \\
\hline $320-660$ & 1 & 1 & 1 & 1 & 1 & 1 & 1 & 1 & 1 & 0 & 1 & 1 & 1 & 0 & 0 & 0 & 1 & 1 & 1 & 0 & 0 & 0 & 0 & 0 & 0 & 0 & 0 & 0 & 0 & 0 & 0 & 0 & 0 & 0 & 0 & 0 \\
\hline $820-650$ & 1 & 1 & 1 & 1 & 1 & 1 & 1 & 1 & 1 & 0 & 1 & 1 & 1 & 0 & 0 & 0 & 1 & 1 & 1 & 0 & 0 & 0 & 0 & 0 & 0 & 0 & 0 & 0 & 0 & 0 & 0 & 0 & 0 & 0 & 0 & 0 \\
\hline $822-750$ & 1 & 1 & 1 & 1 & 1 & 1 & 1 & 1 & 1 & 0 & 1 & 1 & 1 & 0 & 1 & 1 & 1 & 0 & 1 & 0 & 1 & 1 & 1 & 1 & 0 & 0 & 1 & 1 & 0 & 0 & $\cdot 1$ & 0 & 0 & 1 & 1 & 1 \\
\hline $834-575$ & 1 & 1 & 0 & 0 & 1 & 1 & 1 & 1 & 0 & 0 & 1 & 0 & 0 & 0 & 1 & 0 & 1 & 1 & 1 & 0 & 1 & 1 & 0 & 0 & 1 & 0 & 0 & 1 & 0 & 0 & 1 & 0 & 1 & 1 & 0 & 0 \\
\hline $834-375$ & 1 & 1 & 1 & 1 & 1 & 1 & 1 & 1 & 1 & 1 & 0 & 1 & 1 & 1 & 0 & 1 & 0 & 1 & 1 & 1 & 0 & 0 & 1 & 1 & 0 & 1 & 1 & 0 & 0 & 1 & 0 & 1 & 1 & 0 & 1 & 1 \\
\hline $834-325$ & 1 & 1 & 1 & 1 & 1 & 1 & 1 & 1 & 1 & 0 & 1 & 1 & 1 & 0 & 1 & 1 & 1 & 0 & 1 & 0 & 1 & 1 & 0 & 0 & 1 & 0 & 0 & 1 & 1 & 0 & 1 & 1 & 1 & 1 & 1 & 1 \\
\hline $840-1550$ & 0 & 0 & 0 & 0 & 0 & 0 & 0 & 0 & 0 & 1 & 0 & 0 & 0 & 1 & 1 & 0 & 0 & 0 & 0 & 1 & 1 & 1 & 1 & 1 & 1 & 1 & 1 & 1 & 1 & 1 & 1 & 1 & 1 & 1 & 1 & 1 \\
\hline $840-1400$ & 0 & 0 & 0 & 0 & 0 & 0 & 0 & 0 & 0 & 1 & 0 & 0 & 0 & 1 & 1 & 1 & 0 & 0 & 0 & 1 & 1 & 1 & 1 & 1 & 1 & 1 & 1 & 1 & 1 & 1 & 1 & 1 & 1 & 1 & 1 & 1 \\
\hline $840-675$ & 0 & 0 & 0 & 1 & 0 & 0 & 1 & 1 & 0 & 1 & 0 & 0 & 0 & 1 & 1 & 1 & 0 & 0 & 0 & 1 & 1 & 1 & 1 & 1 & 1 & 1 & 1 & 1 & 1 & 1 & 1 & 1 & 1 & 1 & 1 & 1 \\
\hline $841-725$ & 1 & 1 & 1 & 1 & 1 & 1 & 1 & 1 & 1 & 0 & 1 & 1 & 1 & 1 & 1 & 1 & 1 & 1 & 1 & 0 & 0 & 0 & 0 & 0 & 0 & 0 & 0 & 0 & 0 & 0 & 0 & 0 & 0 & 0 & 0 & 0 \\
\hline 843-725 & 1 & 1 & 1 & 1 & 1 & 1 & 1 & 1 & 1 & 0 & 1 & 1 & 1 & 1 & 0 & 1 & 1 & 1 & 1 & 0 & 0 & 0 & 1 & 1 & 1 & 1 & 0 & 1 & 1 & 1 & 1 & 1 & 1 & 1 & 1 & 1 \\
\hline $843-375$ & 1 & 1 & 1 & 1 & 1 & 1 & 1 & 1 & 0 & 0 & 1 & 1 & 1 & 0 & 0 & 0 & 1 & 1 & 1 & 0 & 0 & 1 & 0 & 1 & 1 & 0 & 0 & 0 & 0 & 1 & 0 & 0 & 0 & 0 & 0 & 0 \\
\hline $844-1000$ & 1. & 1 & 1 & 1 & 1 & 1 & 1 & 1 & 0 & 1 & 0 & 1 & 1 & 1 & 0 & 1 & 0 & 0 & 1 & 1 & 1 & 1 & 1 & 1 & 0 & 1 & 1 & 1 & 0 & 1 & 1 & 1 & 1 & 1 & 1 & 1 \\
\hline $844-950$ & 0 & 0 & 0 & 1 & 1 & 1 & 0 & 1 & 0 & 1 & 0 & 1 & 1 & 1 & 0 & 0 & 0 & 0 & 0 & 0 & 1 & 1 & 0 & 0 & 0 & 0 & 0 & 1 & 0 & 0 & 1 & 1 & 1 & 1 & 1 & 0 \\
\hline$\overline{\overline{\text { AFLP }}}$ & & & & & & & & & & & & & & & & & & & & & & & & & & & & & & & & & & & & \\
\hline Markers & AS & As & AG & AP & BD & BS & cs & CG & $\mathrm{cr}$ & CE & $\mathrm{Co}$ & & DL & DQ & DB & FXF & FF & GR & GN & GS C & Gr & GM I & HU & & IS J & JB & Kar & Ks & KD L & LB & MK & MS N & MN I & NM I & NS & PK \\
\hline AAG-CAT- $79^{Y}$ & 0 & 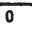 & 1 & 1 & 1 & 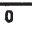 & 1 & 0 & 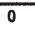 & 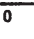 & 0 & 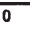 & 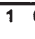 & 0 & $\overline{1}$ & 10 & 0 & 0 & 11 & 1 & 4 & 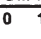 & 1 & 0 & 00 & & 10 & & 0 & 1 & 0 & 0 & & 0 & & 0 \\
\hline AAG-CAT-86 & 0 & 1 & 1 & 1 & 1 & 1 & 1 & 0 & 0 & 0 & 0 & 0 & 1 & 0 & 0 & 10 & 0 & 0 & 10 & 01 & 1 & 0 & 1 & 0 & 0 & & 10 & 0 & 00 & 0 & 0 & 00 & 0 & 0 & 0 & 0 \\
\hline AAG-CAT-110 & 0 & 0 & 0 & 0 & 0 & 0 & 0 & 0 & 0 & 0 & 0 & 0 & 1 & 0 & 0 & 0 & 0 & 0 & 01 & 1 & 1 & 0 & 0 & 0 & 00 & & 10 & 0 & 0. & 1 & 0 & 00 & 0 & 1 & 0 & 0 \\
\hline AAG-CAT-118 & 0 & 1 & 0 & 0 & 0 & 0 & 0 & 0 & 0 & 0 & 0 & 0 & 0 & 0 & 0 & 10 & 0 & 0 & 00 & 00 & 0 & 0 & 0 & 0 & 00 & & 00 & 0 & 01 & 1 & 0 & 00 & 0 & 0 & 0 & 0 \\
\hline AAG-CAT-119 & 0 & 0 & 1 & 1 & 0 & 1 & 1 & 0 & 1 & 0 & 0 & 1 & 1 & 0 & 0 & 0 & 0 & 0 & 10 & 0 & 1 & 0 & 1 & 0 & 10 & & 10 & 0 & 00 & 0 & 0 & 00 & 0 & 0 & 0 & 0 \\
\hline AAG-CAT-163 & 0 & 0 & 1 & 0 & 0 & 0 & 0 & 0 & 0 & 0 & 0 & 0 & 1 & 1 & 0 & 00 & 0 & 0 & 00 & 0 & 0 & 0 & 0 & 1 & 00 & & 00 & 0 & 00 & 0 & 0 & 10 & 0 & 1 & 0 & 0 \\
\hline AAG-CAT-169 & 0 & 1 & 1 & 1 & 1 & 1 & 1 & 0 & 1 & 0 & 0 & 1 & 1 & 0 & 0 & 10 & 0 & 1 & 10 & 0 & 1 & 0 & 1 & 1 & 00 & & 01 & 1 & 01 & 1 & 0 & 00 & 0 & 0 & 0 & 0 \\
\hline AAG-CTC-83 & 0 & 0 & 0 & 0 & 1 & 0 & 0 & 0 & 0 & 0 & 0 & 0 & 0 & 1 & 0 & 0 & 0 & 0 & 0 & 0 & 0 & 0 & 1 & 0 & 00 & 0 & 10 & 0 & 10 & 0 & 0 & 00 & 0 & 0 & 0 & 0 \\
\hline AAG-CTC-111 & 0 & 0 & 1 & 1 & 0 & 0 & 0 & 0 & 0 & 0 & 1 & 0 & 0 & 0 & 0 & 0 & 0 & 1 & 10 & 0 & 1 & 0 & 0 & 0 & 00 & & 10 & 0 & 0 & 0 & 0 & 00 & 0 & 0 & 0 & 0 \\
\hline AAG-CTC-123 & 0 & 0 & 1 & 0 & 1 & 0 & 0 & 0 & 0 & 0 & 1 & 0 & 0 & 0 & 0 & 0 & 0 & 0 & 11 & 1 & 1 & 0 & 1 & 0 & 00 & 0 & 00 & 0 & 1 & 0 & 0 & 00 & 0 & 0 & 0 & 0 \\
\hline AAG-CTC-130 & 0 & 1 & 1 & 1 & 1 & 1 & 0 & 0 & 0 & 0 & 1 & 1 & 0 & 0 & 0 & 1 & 0 & 1 & 1 & 0 & 1 & 0 & 1 & 0 & 00 & & 00 & 0 & 0 & 0 & 0 & 00 & 0 & 0 & 0 & 0 \\
\hline AAG_CTC-137 & 0 & 0 & 0 & 0 & 0 & 0 & 0 & 0 & 0 & 0 & 0 & 0 & 0 & 0 & 0 & 0 & 0 & 0 & 0 & 0 & 1 & 0 & 1 & 0 & 00 & & 00 & 0 & 10 & 0 & 0 & 00 & 0 & 0 & 0 & 0 \\
\hline AAG-CTC-138 & 0 & 1 & 1 & 1 & 1 & 1 & 0 & 0 & 0 & 0 & 1 & 1 & 0 & 0 & 0 & 1 & 0 & 1 & 1 & 0 & 0 & 0 & 0 & 0 & 00 & 0 & 00 & 0 & 0 & 0 & 0 & 00 & 0 & 0 & 0 & 0 \\
\hline G-CTC & 0 & 0 & 1 & 1 & 1 & 0 & 0 & 0 & 0 & 0 & 0 & 0 & 0 & 0 & 0 & 0 & 0 & 0 & 0 & 0 & 0 & 0 & 0 & 0 & 0 & 0 & 00 & & 0 & 0 & 0 & 0 & 0 & 0 & 0 & 0 \\
\hline AAG-CTC-206 & 0 & 1 & 0 & 1 & 0 & 0 & 0 & 0 & 0 & 0 & 0 & 0 & 0 & 0 & 0 & 0 & 0 & 0 & 0 & 1 & 0 & 0 & 0 & 0 & 0 & & 00 & & 0 & & 0 & & & & 0 & \\
\hline AMG-CTC-207 & 0 & 0 & 1 & 0 & 1 & 1 & 0 & 0 & 0 & 0 & 1 & 0 & 0 & 0 & 0 & 0 & 0 & 1 & 0 & 0 & 0 & 0 & 1 & 0 & 0 & 0 & 00 & 0 & 1 & 0 & 0 & 0 & 0 & 0 & 0 & 0 \\
\hline AAG-CTC-221 & 0 & 1 & 0 & 0 & 1 & 1 & 0 & 0 & 0 & 0 & 0 & 1 & 0 & 0 & 0 & 0 & 0 & 0 & 0 & 0 & 0 & 0 & 1 & 0 & 00 & & 00 & 0 & 0 & 0 & 0 & 0 & 0 & 0 & 0 & 0 \\
\hline AAG-CTC-315 & 0 & 0 & 0 & 1 & 1 & 0 & 0 & 0 & 0 & 1 & 1 & 0 & 0 & 0 & 0 & 0 & 0 & 0 & 0 & 0 & 0 & 0 & 0 & 0 & 0 & 0 & 00 & 0 & 0 & 0 & 0 & 00 & 0 & 0 & 0 & 0 \\
\hline AMG-CTC-453 & 0 & 1 & 0 & 1 & 1 & 0 & 0 & 0 & 0 & 0 & 1 & 1 & 0 & 0 & 0 & 1 & 0 & 1 & 1 & 0 & 0 & 0 & 0 & 0 & 0 & 0 & 0 & 0 & 0 & 0 & 0 & 0 & 0 & 0 & 0 & 0 \\
\hline 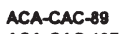 & & 1 & 0 & 1 & 1 & 1 & 1 & & 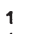 & 0 & 0 & 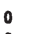 & 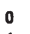 & 0 & 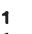 & 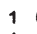 & 0 & & 1 & 0 & 1 & 0 & 1 & 1 & 1 & 0 & 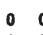 & & & & & & & & & \\
\hline CAC- & 1 & 1 & 1 & 1 & 1 & 1 & 1 & & 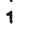 & 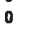 & 0 & 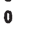 & 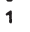 & 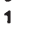 & 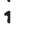 & 1 & 1 & 0 & I & & 1 & 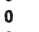 & 1 & 0 & & & & & & 0 & & & & & & \\
\hline CAC & . & 1 & 0 & 1 & 1 & 1 & 1 & & 1 & 0 & 0 & 0 & 0 & 0 & 0 & 1 & 1 & 0 & $i$ & 0 & 1 & 0 & 0 & 0 & 0 & & & & & 0 & & & & & & \\
\hline & 0 & 0 & 0 & 0 & 1 & 1 & 0 & & 1 & 0 & 0 & 0 & . & 0 & 0 & & & & 0 & & & & 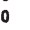 & 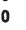 & & & & & & & & & & & & \\
\hline$A c$ & 0 & 1 & 0 & 1 & $i$ & 1 & 1 & (19. & 1 & 0 & 0 & 0 & 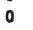 & 0 & 0 & 1 & 1 & 0 & 1 & 0 & 0 & 0 & 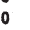 & 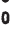 & 0 & & & & & 0 & & 0 & & & 0 & 0 \\
\hline AT & 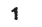 & 1 & 0 & 1 & 0 & 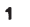 & 0 & & 0 & 0 & 0 & 0 & 0 & 0 & 0 & 1 & & & 0 & 0 & 0 & 1 & 0 & 0 & 0 & & 0 & & 1 & & & & & & & \\
\hline & 0 & 0 & 0 & 0 & 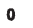 & 0 & 0 & & o & 0 & 0 & 0 & & 0 & 0 & & & & & & & & & & & & & & & & & & & & & \\
\hline TT & 1 & 1 & 0 & 0 & 0 & 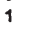 & 0 & 0 & 0 & 0 & 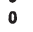 & 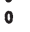 & 1 & 1 & 1 & 0 & 0 & 0 & 0 & 1 & 0 & 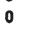 & & & & & 0 & & & & & & & & & \\
\hline C-CAT-90 & 1 & 1 & 0 & 1 & 1 & 1 & 0 & 1 & 0 & 1 & 0 & 0 & 1 & 0 & 0 & 1 & 1 & 1 & 1 & 0 & 1 & 1 & 1 & 0 & 00 & & 0 & & 1 & 1 & & & & & 1 & \\
\hline ACC-CAT-91 & 0 & 0 & 0 & 0 & 0 & 0 & 0 & 0 & 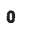 & 0 & 0 & 0 & 0 & 1 & 0 & & & & 0 & & 0 & 0 & 0 & 0 & & & 0 & & 0 & 0 & & & & & 0 & \\
\hline & 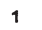 & 1 & 0 & 1 & 1 & 1 & 0 & 1 & 0 & 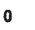 & 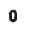 & 0 & 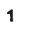 & & 1 & & & & & & & & & & & & & & & & & & & & & \\
\hline & 0 & 0 & 0 & 0 & 0 & 0 & 0 & 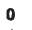 & 0 & 0 & 0 & 0 & 0 & 1 & 0 & & 0 & 0 & 0 & & 0 & 0 & 0 & 0 & & & & & & & & & & & & \\
\hline C-CAT-122 & 1 & 1 & 0 & 1 & 1 & 1 & 0 & 1 & 0 & 0 & 0 & 0 & 1 & 1 & 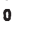 & 1 & 1 & 0 & 1 & 0 & 1 & 0 & 1 & 0 & & & 0 & 0 & 0 & 0 & 0 & 0 & & & 0 & 0 \\
\hline & 1 & 1 & 0 & 0 & 0 & $\begin{array}{l}1 \\
0\end{array}$ & 0 & 0 & $\begin{array}{l}0 \\
0\end{array}$ & 0 & 0 & & & 1 & 0 & $\begin{array}{l}0 \\
0\end{array}$ & $\begin{array}{l}0 \\
0\end{array}$ & & 0 & & & & & & $\begin{array}{l}0 \\
0\end{array}$ & & & & & & & & & & & \\
\hline
\end{tabular}


for DNA transcription at $72{ }^{\circ} \mathrm{C}$, followed by a cycle of $120 \mathrm{~s}$ at $94{ }^{\circ} \mathrm{C}$ to denature DNA, $30 \mathrm{~s}$ for DNA annealing at $64{ }^{\circ} \mathrm{C}$, and $120 \mathrm{~s}$ for DNA transcription at $72{ }^{\circ} \mathrm{C}$. The second cycle was followed by seven cycles of same conditions, but with only 20 $\mathrm{s}$ at $94^{\circ} \mathrm{C}$ to denature DNA, and the annealing temperature was gradually reduced by $1{ }^{\circ} \mathrm{C}$ in each sequential cycle from $63^{\circ} \mathrm{C}$ at the first cycle to $57^{\circ} \mathrm{C}$ at the seventh cycle. The second part of the selective amplification consisted of 25 cycles of $20 \mathrm{~s}$ at $94{ }^{\circ} \mathrm{C}$ to denature DNA, $30 \mathrm{~s}$ for DNA annealing at $56{ }^{\circ} \mathrm{C}$, and $120 \mathrm{~s}$ at $72^{\circ} \mathrm{C}$ for DNA transcription, followed by $30 \mathrm{~min}$ at 60 ${ }^{\circ} \mathrm{C}$ for any additional annealing and DNA transcription, and an extended cooling period at $4{ }^{\circ} \mathrm{C}$.

Reactions were prepared for electrophoresis analysis by mixing $1 \mu \mathrm{L}$ of the final selective amplification reaction with $1.2 \mu \mathrm{L}$ of formamide : blue dextran $\left(30 \mathrm{mg} \cdot \mathrm{L}^{-1}\right)$ (mixed in a ratio of $\left.5: 1\right)$, and $0.3 \mu$ Lof ROX 500 Genescan standard (Applied Biosystems). Samples $(2.2 \mu \mathrm{L})$ were loaded on a $6 \%$ polyacrylamide gel, and electrophoresed at $3000 \mathrm{~V}$ using a Perkin Elmer ABI-373 or ABI377 Sequencer (according to manufacturer's protocol). Fragment sizes were determined using Genescan and Genotyper software (Applied Biosystems).

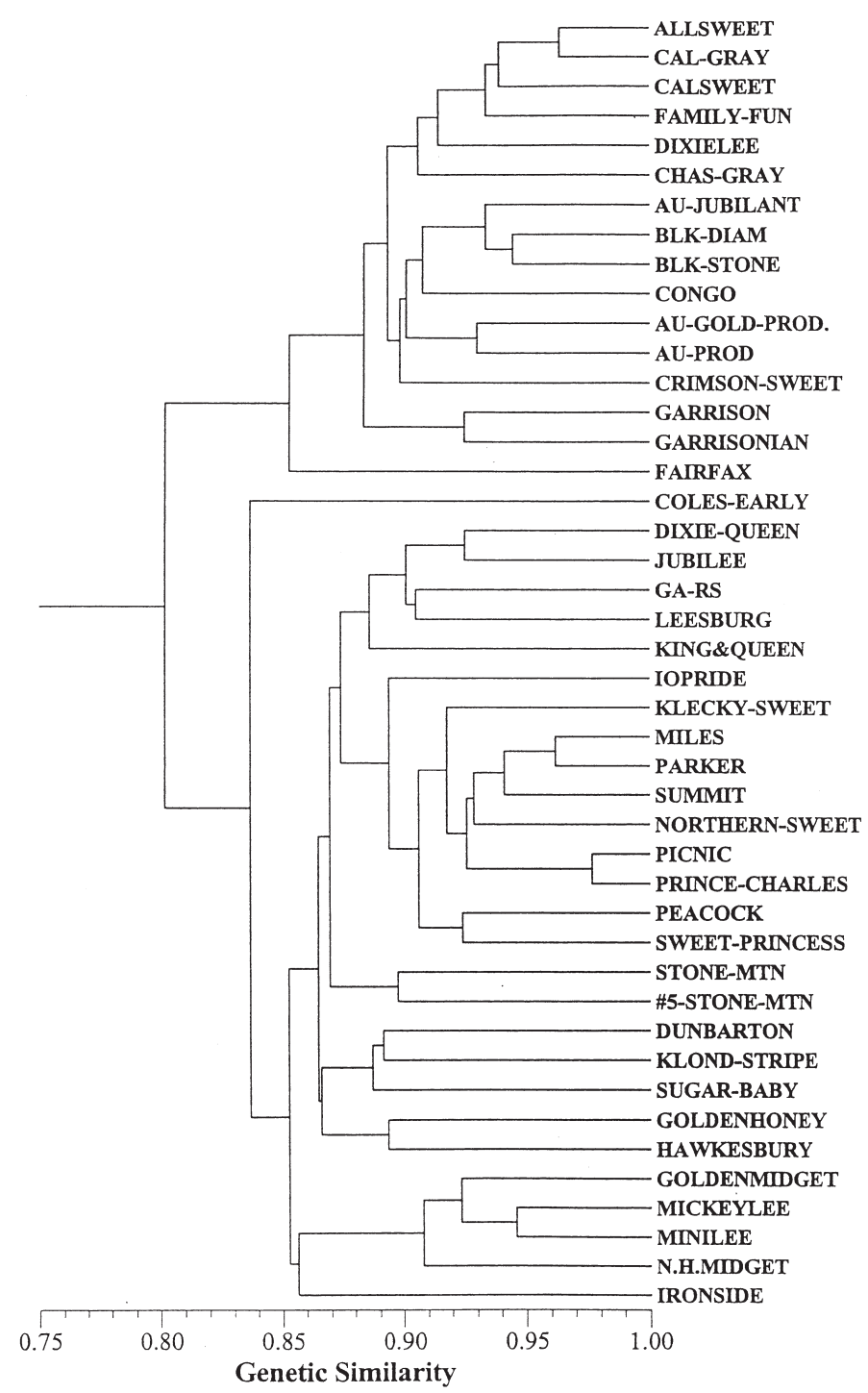

Fig. 2. Dendogram of watermelon cultivars produced by UPGMA cluster analysis of similarity matrix based on 229 ISSR and AFLP markers.
Data ANALYsis. A pairwise similarity matrix was generated using the Nei-Li similarity index (Nei and Li, 1979) according to the equation: Similarity $=2 \mathrm{~N}_{\mathrm{ab}} /\left(\mathrm{N}_{\mathrm{a}}+\mathrm{N}_{\mathrm{b}}\right)$, Where $\mathrm{N}_{\mathrm{ab}}$ is the number of fragments (ISSRs and AFLPs) shared by two genotypes (a and $\mathrm{b}$ ) and $\mathrm{N}_{\mathrm{a}}$ and $\mathrm{N}_{\mathrm{b}}$ are the total number of fragments analyzed in each genotype. A dendrogram was constructed based on the similarity matrix data by applying the unweighted pair-group method with arithmetic average (UPGMA) cluster analysis using the Numerical Taxonomic and Multi-Variant Analysis System for PC (NTSYS-PC version 2) (Rohlf, 1993).

\section{Results and Discussion}

ISSR MARKERS. The ISSR markers identified significant polymorphisms and were consistent among experiments due to optimization of the annealing temperature in PCR for each of the ISSR primers (Table 2). Thirty-one (81.6\%) of the 38 ISSR primers produced polymorphic markers. Seventy-two (64.5\%) of 111 ISSR markers were polymorphic among cultivars (Table 2, Fig. 1). Previously Levi et al. (2001) observed only 85 (29.5\%) of 288 RAPD markers that were polymorphic among the same cultivars. ISSRs exhibited more polymorphisms than RAPD markers in various plant species, as shown in experiments with rice varieties (Davierwala et al., 2000) and with Finger millet (Eleusine coracana) accessions (Salimath et al., 1995). Simple sequence repeats are present throughout the genome and are likely to be clustered near unique gene sequences (Temnykh et al., 2001; Wang et al., 2002). ISSR markers have been used successfully to detect polymorphisms in crop plants as shown for cauliflower (Bornet et al., 2002), fruit plants including strawberry, apple and Ribes species (Korbin et al., 2002) and in Cucurbita pepo (Paris et al., 2003). Bornet et al. (2002) showed that ISSR primers were useful in detecting simple sequence repeat regions across the Brassica oleracea genome, and that many ISSR-internal regions had homologies with known gene sequences (mainly with genes coding for proteins implicated in DNA interaction or gene expression).

AFLP MARKERS. All AFLP fragments produced by the four EcoRI / MseI primer pairs in this study were polymorphic among cultivars. AFLP primer pairs 1, 2, 3, and 4 produced 35, 33, 26, and 24 polymorphic markers, respectively (representative markers are shown in Table 3). Overall, the AFLP markers in this study appeared to be highly polymorphic among watermelon cultivars compared with ISSR and RAPD markers. Ke-peng et al. (2003) examined genetic diversity among watermelon accessions developed in China using eight selective AFLP primer pairs different from those used in this study. In that study, the introduction of EcoRI selective primer with two nucleotides at the 3' end (EcoRI-AT and MseI-CTA, EcoRI-AT and MseI-CAT,EcoRI-AA and MseI-CAA, EcoRI-AC and MseI-CTT, and EcoRI-AG and MseI-CAA) resulted in producing large numbers of AFLP fragments (94, 120 102, 92, 76 , and 87 fragments, respectively). However, in contrast with the results in this study, not all AFLP fragments were polymorphic among the Chinese accessions. These differences might be due to the higher resolution using a DNA sequencer (ABI 373, Applied Biosystems) in this study, versus visual reading of AFLP gels as was performed for the Chinese accessions (Ke-peng et al., 2003). AFLPs are efficient in detecting polymorphisms in crop plants with limited genetic diversity as shown with cotton (Abdalla et al., 2001), cowpea landraces (Tosti and Negri, 2002), and cashew accessions (Archak et al., 2003). Bonnema et al. (2002) indicated that AFLP markers may cover wider genomic regions of tomato 
that are not readily covered by RFLP markers.

EFFECTIVE USE OF MOLECULAR MARKERS IN WATERMELON BREEDING PROGRAMS. A commonly held view is that cultivated watermelon has a narrow genetic base due to recurrent selection and continuous inbreeding during 5000 years of cultivation (Navot and Zamir, 1987). The narrow genetic base is notably reflected in no or low isozyme polymorphisms among watermelon cultivars and accessions (Navot and Zamir, 1987). In a previous study (Levi et al., 2001), 288 RAPD markers produced low polymorphisms (92.5\% to $98.9 \%$ genetic similarity) and could hardly differentiate among some of the heirloom cultivars. In this study, a comparable number of ISSR and AFLP markers (altogether 229) produced higher polymorphisms (80.2\% to $97.8 \%$ genetic similarity) among all heirlooms. Also, the genetic similarity values among cultivars (Table 1) and their phylogenetic relations (Fig. 2) are consistent with the parental records collected by Elmstrom (1999) (Table 1), e.g., 'Garrison' and 'Garrisonian', 'AU-Producer' and 'AUGolden Producer', 'Mickylee' and 'Minilee', 'Black-Diamond' and 'Black Stone', and 'Stone Mountain' and 'Stone Mountain \#5'. Based on parental information (Elmstrom, 1999) the cultivars in each pair are closely related as shown in the dendrogram (Fig. 2). ISSRs and AFLPs efficiently differentiate among watermelon heirloom cultivars with limited genetic diversity. It is apparent that AFLP technology is more effective than ISSRs and RAPDs in identifying closely related breeding lines of watermelon, and in identifying triploid (seedless) watermelons derived from closely related genotypes. The large number of polymorphic ISSR and AFLP markers also provide better resolution with respect to relatedness among watermelon cultivars as compared with RAPD markers. Conversion of specific AFLP or ISSR fragments to sequenced characterized amplified region (SCAR) markers could enhance the value of these markers for watermelon cultivar identification. Furthermore, AFLP markers appeared to be clustered and cover linkage regions different from ISSR and RAPD markers, as recently shown in a linkage map constructed for melon (Cucumis melo) (Perin et al., 2002) and in our mapping experiments for watermelon (A. Levi, unpublished data). Thus, AFLP, ISSR and RAPD markers should complement each other in genetic identification and in covering different regions of watermelon genome.

\section{Literature Cited}

Abdalla, A.M., O.U.K. Reddy, K.M. El-Zik, and A.E. Pepper. 2001. Genetic diversity and relationships of diploid and tetraploid cottons revealed using AFLP. Theor. Appl. Genet. 102:222-229.

Archak, S., A.B. Gaikwad, D. Gautam, E.V. Rao, K.R. Swamy, and J.L. Karihaloo. 2003. Comparative assessment of DNA fingerprinting techniques (RAPD, ISSR and AFLP) for genetic analysis of cashew (Anacardium occidentale L.) accessions of India. Genome 46:362-369.

Bonnema, G., P. van den Berg, and P. Lindhout. 2002. AFLPs mark different genomic regions compared with RFLPs: A case study in tomato. Genome 45:217-221.

Bornet, B., C. Muller, F. Paulus, and M. Branchard. 2002. Highly informative nature of inter simple sequence repeat (ISSR) sequences amplified using tri- and tetra-nucleotide primers from DNA of cauliflower (Brassica oleracea var. botrytis L.). Genome 45:890-896.

Davierwala, A.P., K.V. Chowdari, S. Kumar, A.P. Reddy, P.K. Ranjekar, and V.S. Gupta. 2000. Use of three different marker systems to estimate genetic diversity of Indian elite rice varieties. Genetica 108:269-84.

Elmstrom, G.W. 1999. Vegetable cultivar descriptions for North America. HortScience 34:1010-1012.

Germplasm Resources Information Network (GRIN). 2003. Online database, National Germplasm Resources Laboratory, USDA, ARS, Beltsville, Md. 2003. Evaluation/characterization Data Queries. Retrieved 14 Dec. 2002 from http://www.ars-grin.gov/cgi-bin/npgs/ $\mathrm{html} /$ croplist.pl.

Ke-peng, C., C. Liang, Y. Wang, D. Jin, B. Wang, Y. Xu, G. Kang, and H. Zhang. 2003. Genetic assessment of watermelon germplasm using the AFLP technique. HortScience 38:81-84.

Korbin, M., A. Kuras, and E. Zurawicz. 2002. Fruit plant germplasm characterization using molecular markers generated in RAPD and ISSR-PCR. Cell Mol. Biol. Lett. 7:785-794.

Levi, A., L.J. Rowland, and J.S. Hartung. 1993. Production of reliable randomly amplified polymorphic DNA (RAPD) markers from DNA of woody plants. HortScience 28:1188-1190.

Levi, A. and C.E. Thomas. 1999. An improved procedure for isolation of high quality DNA from watermelon and melon leaves. Cucurbit Genet. Coop. Rpt. 22:41-42.

Levi, A., C.E. Thomas, T.C. Wehner, and X.P. Zhang. 2001. Low genetic diversity indicates the need to broaden the genetic base of cultivated watermelon. HortScience 36:1096-1101.

Navot, N. and D. Zamir. 1987. Isozyme and seed protein phylogeny of the genus Citrullus (Cucurbitaceae). Plant Syst. Evol. 156:61-67.

Nei, M. and W. Li. 1979. Mathematical model for studying genetic variation in terms of restriction endonucleases. Proc. Natl. Acad. Sci. USA 76:5269-5273.

Paris, H.S., N. Yonash, V. Portnoy, N. Mozes-Daube, G. Tzuri and N. Katzir. 2003.

Assessment of genetic relationships in Cucurbita pepo (Cucurbitaceae) using DNA markers. Theor. Appl. Genet. 106:971-978.

Perin, C., L.S. Hagen, V. De Conto, N. Katzir, Y. Danin-Poleg, V. Portnoy, S. Baudracco-Arnas, J. Chadoeuf, C. Dogimont, and M. Pitrat. 2002. A reference map of Cucumis melo based on two recombinant inbred line populations. Theor. Appl. Genet. 104:1017-1034.

Rohlf, F.J. 1993. NTSYS-PC numerical taxonomy and multivariate analysis system. version 2.00. Exter Publ., Ltd., Setauket, N.Y.

Salimath, S.S., A.C. de Oliveira, I.D. Godwin, and J.L. Bennetzen. 1995. Assessment of genome origins and genetic diversity in the genus Eleusine with DNA markers. Genome 38:757-763.

Sambrook, J., E.F. Fritsch, and T. Maniatis. 1989. Molecular cloning. A laboratory manual. 2nd ed. Cold Spring Harbor Laboratory Press, Plainview, N.Y.

Temnykh, S., G. DeClerck, A. Lukashova, L. Lipovich, S. Cartinhour, and S. McCouch. 2001. Computational and experimental analysis of microsatellites in rice (Oryza sativa L.): Frequency, length variation, transposon associations, and genetic marker potential. Genome Res. 11:1441-1152.

Tosti, N. and V. Negri. 2002. Efficiency of three PCR-based markers in assessing genetic variation among cowpea (Vigna unguiculata subsp. unguiculata) landraces. Genome 45:268-275.

U.S. Department of Agriculture. 2003. Agricultural statistics. U.S. Govt. Printing Office, USDA, Wash., D.C.

Vos, P., R. Hogers, M. Beeker, M. Reijans, T. Vandelee, M. Hornes, A. Frijiters, J. Pot, J. Peleman, M. Kuiper, and M. Zabeau. 1995. AFLP - A new technique for DNA fingerprinting. Nucl. Acids Res. 23:4407-4414.

Wang, Y., L.L. Georgi, T.N. Zhebentyayeva, G.L. Reighard, R. Scorza, and A.G. Abbott. 2002. High-throughput targeted SSR marker development in peach (Prunus persica). Genome 45:319-28. 